\title{
Adolescents with greater mental toughness show higher sleep efficiency, more deep sleep and fewer awakenings after sleep onset
}

Serge Brand, $\mathrm{PhD}^{1 * \#, ~ M a r k u s ~ G e r b e r, ~} \mathrm{PhD}^{2 *}$, Nadeem Kalak, $\mathrm{MSc}^{1}$, Roumen Kirov, $\mathrm{MD}, \mathrm{PhD}^{3}$, Sakari Lemola, $\mathrm{PhD}^{4}$, Peter J. Clough, $\mathrm{PhD}^{5}$, Uwe Pühse, $\mathrm{PhD}^{2}$, and Edith Holsboer-Trachsler, $\mathrm{MD}^{1}$

${ }^{1}$ Psychiatric Hospital of the University of Basel, Basel, Switzerland

${ }^{2}$ Institute of Exercise and Health Sciences, University of Basel, Basel, Switzerland

${ }^{3}$ Institute of Neurobiology, Bulgarian Academy of Sciences, Sofia, Bulgaria

${ }^{4}$ Department of Psychology, University of Basel, Basel, Switzerland

${ }^{5}$ Department of Psychology, University of Hull, Hull, United Kingdom

All authors declare no conflicts of interests, and the study has been conducted without external funding.

* Both these authors contributed equally

\# Correspondence

Serge Brand, Ph.D.

Psychiatric Clinics of the University of Basel

Center for Affective, Stress and Sleep Disorders

Wilhelm Klein-Strasse 27

4012 Basel - Switzerland

+41613255114 (voice)

+41613255 513 (fax)

serge.brand@upkbs.ch (email)

\section{Acknowledgements}


We thank Nick Emler (Surrey, UK) for proofreading the manuscript.

Moreover, we thank Marielle Koenig and Vladimir Djurdjevic from the sleep lab of the Center for Affective, Stress and Sleep Disorders for sleep-EEG recordings and analysis. 


\section{Background: To investigate the association between mental-}

toughness and-objectively assessed-steep-Mental toughness (MT) is understood as the display of confidence, commitment, challenge and control. MT is associated with greater resilience against stress. There is as yet, however, no research focusing on the relation between MT and objective sleep. The aim of the present study was therefore to explore the extent to which greater MT is associated with objectively assessed sleep among adolescents.

Method: A total of 92 adolescents (35\% females; mean age: $M=18.92$ years) completed the Mental Toughness Questionnaire (MTQ48). Participants were split into a group of high (H-MT) and into a group of low mental toughness (L-MT). Moreover, o-Objective sleep was recorded via sleep-EEGs, and subjective sleep was assessed with a

\section{questionnaire.}

Results: Compared to participants with low MT, participants with high MT had higher sleep efficiency, a lower number of awakenings after sleep onset, less light sleep, and more deep sleep. They also reported lower daytime sleepiness.

Conclusions: Adolescents reporting higher mental toughness also had objectively better sleep, as recorded via sleep-EEG. A bi-directional association between mental toughness and sleep seems likely; therefore, among adolescence, improving sleep should increase MT, as also improving MT should increase sleep.

Key-words: mental toughness; sleep-EEG, sleep efficiency, deep sleep 
Implications and Contribution

Mentally tough adolescents showed objectively assessed improved sleep. Improving adolescents' mental toughness might also improve adolescents' sleep and vice versa.

Introduction

Adolescence is defined as the period of gradual transition between childhood and adulthood, with conceptually distinct physical changes marking puberty and maturation $[1,2]$. Along with dramatic changes in physiology and neural networks $[3,4]$, adolescents have to face new challenges and assume responsibility for issues such as their academic and vocational careers, peer and intimate relationships, increased physical, emotional and financial independence from parents and siblings, use of psychoactive substances, extra-curricular employments and leisuretime activities such as sports participation and music (see [1] for extensive overview). Dealing with these issues is potentially stressful and, accordingly it is assumed that adolescents with better coping skills will deal more successfully with these challenges [5].

A psychological construct related to favorable stress management is mental toughness (MT). Mental toughness (MT) is a relatively new area of academic research [6], and a cognitive strength variable known to be associated with good performance both in elite sport [7] and, more recently, also in non-elite sport [8-11]. Mental toughness has been conceptualized in various ways in the scientific literature (see [6] for review). In the present study, we used the $4 C(+2)$ model of mental 
toughness defined as performing well in challenging situations ('Challenges usually bring out the best in me.'), commitment ('I don't usually give up under pressure.'), control [emotional control: 'Even when under considerable pressure I usually remain calm.'; life control: ‘l generally feel in control.') and confidence [interpersonal confidence: 'I usually take charge of a situation when I feel it is appropriate.'; confidence in ability: 'I am generally confident in my own abilities.']; cf. [12]). In previous studies [9-11] we have been able to validate a German version of the Mental Toughness Questionnaire 48 (MTQ48; [12]) and to show, in a large sample of adolescents and young adults, 1) that the construct of mental toughness (MT) is not limited to high performing elite sportsmen and women $[9-11], 2)$ that MT is associated with increased stress resilience $[9,10]$, and 3 ) that MT remains stable over time [11], suggesting therefore that MT is related to successful stress management and to psychological well-being.

With regard to this last construct, numerous studies have indicated that, independent of age, there is a bi-directional relation between psychological well-being and sleep [13]. For adolescents, sleep and sleep regulation play a crucial role in both well-being and development [14-17]. To illustrate, Lemola et al $[18,19]$ were able to show that dispositional optimism was associated with better sleep quality and longer sleep duration among children and adults. By contrast, sleep disturbances have been reported in more than $25 \%$ of adolescents worldwide; poor sleep in adolescence has become a significant public mental and physical health problem $[14,20]$. Cross-sectional $[21,22]$ and longitudinal studies $[23,24]$ 
have shown that acute and chronic sleep loss during development persists over time, with negative effects on adolescents' physical and mental health. At the same time poor psychological well-being may itself negatively impact on adolescents' sleep $[25,14]$.

To explain the association between poor sleep and psychological processes, it has been proposed that increased arousal and dysfunctional thoughts are directly involved in psychologically caused sleep disturbances [26-29], whereas, for instance, the absence of stress and worries are associated with favorable sleep. In sum, the research reviewed above points to interrelations between low stress, favorable personality traits and restorative sleep. Consequently, it seems possible that high MT and good quality sleep will prove to be closely linked.

Accordingly, the main goal of the research reported here was to explore the association between mental toughness and objective sleep within a sample of adolescents. We believe that the present study can add to the current literature on mental toughness and sleep in an important way in showing a close association between MT (as a marker of psychological well-being) and sleep among non-elite sport adolescents.

The following hypothesis was formulated. Following previous research (Lemola et al $[18,19]$, Gerber et al [9-11]) we expected that higher scores for MT would be associated with improved sleep, as assessed by sleep-EEG recordings. More specifically, we expected a higher sleep efficiency, a shorter sleep onset latency, more deep sleep and less light sleep in adolescents with high MT, as compared to adolescents with low MT. We also expected lower daytime sleepiness. 
Sample

First, a total of 285 adolescents participated in the study (mean age 18.26 years, $\mathrm{SD}=4.17)$ and preliminary date were presented elsewhere [30]. Here, we report data from a subsample: After completing the Mental Toughness Questionnaire (MT; see below), a subsample of participants with high MT scores (upper 17\% of the total score: 141 points and higher) and participants with low MT scores (lower $15.5 \%$ of the total score: 77 points and lower) also completed a questionnaire related to sleep complaints and daytime sleepiness (see below) and underwent further sleep-EEG assessment (see below).

To recruit participants, the study was advertised electronically on the homepages of three high schools in the canton of Basel, a district of the German-speaking Northwestern part of Switzerland. Data were collected during spring and summer 2012. Participants were informed about the purpose of the study and about the voluntary basis of their participation. They were also assured of the confidentiality of their responses, and written informed consent. For children aged below 18 years informed consent was secured from their parents. Participants undergoing sleepEEG recordings received a voucher of 30 Swiss francs (about 30 USD) for participation. The study was approved by the local ethics committee, and the entire study was performed in accordance with the ethical standards laid down in the Declaration of Helsinki.

Materials 
Participants completed the Mental Toughness Questionnaire (MTQ48), the Insomnia Severity Index (ISI), the Epworth Sleepiness Scale (ESS), and underwent objective sleep-EEG monitoring.

Mental toughness

Participants were asked to fill in the 48-item Mental Toughness Questionnaire (MTQ48; [12]; German version: [9-11]), which measures overall mental toughness and its six subcomponents: challenge (e.g. 'Challenges usually bring out the best in me.'), commitment (e.g. 'I don't usually give up under pressure.'), emotional control (e.g. 'Even when under considerable pressure I usually remain calm.'), life control (e.g. 'I generally feel in control.'), interpersonal confidence (e.g. 'I usually take charge of a situation when I feel it is appropriate.') and ability confidence (e.g. 'I am generally confident in my own abilities.'). Answers on the MTQ48 were given on five-point Likert-type scales ranging from 1 (=strongly disagree) to 5 (=strongly agree). Items were summed to obtain overall and subscale scores, with higher scores reflecting greater MT (Cronbach's alpha $=.89$ ).

Assessment of sleep; objective sleep assessment

To assess sleep objectively, Sleep-EEG recordings were performed at home using a three-channel EEG device (Fp2-A1, C3-A2, C4-A1; electrooculogram; electromyogram; Somnowatch ${ }^{\circledR}$; Randersacker, Germany). Sleep polygraphs were visually analyzed by two experienced raters according to the standard procedures [31] (inter-rater reliability: 
kappa $=.88)$. Raters were completely blinded with respect to participants' group assignments. The device provides assessment of total sleep time (TST), sleep efficiency (SE), sleep onset latency (SOL), stages 1-4 (min and $\%$ ), light sleep (stages $1+2$ ), slow wave sleep (stages 3+4), REM-sleep, and number and times of awakenings after sleep onset.

Assessment of sleep; subjective sleep assessment

The Insomnia Severity Index (ISI [32]) is a 7-item screening measure for insomnia and an outcome measure for use in treatment research. The items, answered on 5 -point rating scales ( $0=$ not at all, $4=$ very much), refer in part to DSM-IV (Diagnostic and Statistical Manual of Mental Disorders) criteria for insomnia [33] by measuring difficulty in falling asleep, difficulties remaining asleep, early morning awakenings, increased daytime sleepiness, impaired daytime performance, low satisfaction with sleep, and worrying about sleep. The higher the overall score, the more the respondent is assumed to suffer from insomnia (Cronbach's alpha $=.92$ ).

Daytime sleepiness

To assess daytime sleepiness, participants completed the Epworth Sleepiness Scale (ESS, [34]). Participants were asked about the likelihood of dozing off or falling asleep in specific situations (sitting and reading, watching TV, sitting inactive in a public place, as a passenger in a car for an hour without a break, lying down to rest in the afternoon, sitting and talking to someone, sitting quietly after a lunch). Answers are given on 4- 
pointLikert scales ranging from 0 (=no chance of dozing) to 3 (=high chance of dozing), with a higher sum score reflecting a greater likelihood of dozing off.

Statistical analysis

With a series of single t-tests sleep parameters (sleep-EEG, Insomnia Severity Index, Epworth Sleepiness Scale) between participants with high MT and low MT were compared. Next, to calculate the association between the dimensions of MT and objective sleep parameters, subjective sleep and daytime sleepiness across the entire sample, a series of Pearson's correlations was calculated.

The level of significance was set at alpha $\leq .05$. All statistical analyses were calculated with SPSS ${ }^{\circledR} 19.0$ for Windows.

Results

High and low mental toughness, objective sleep parameters, subjective sleep and daytime sleepiness

Table 1 shows the descriptive and statistical comparisons of sleep parameters, subjective sleep and daytime sleepiness scores between participants with high MT and low MT.

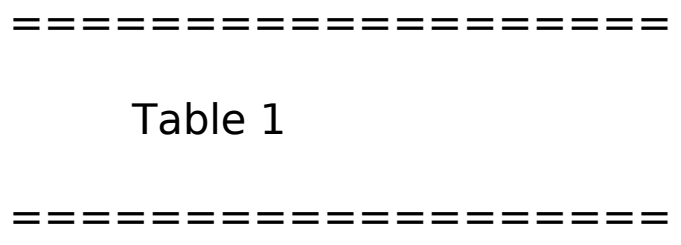


Relative to participants with low MT, participants with high MT had a higher sleep efficiency, more deep sleep, more REM-sleep, less light sleep, less awakenings after sleep onset and a lower score of subjective sleep complaints (ISI) daytime sleepiness. No statistically significant differences were found for sleep duration, sleep onset latency and stage 2 .

Associations between the dimensions of mental toughness and objective sleep dimensions, subjective sleep and daytime sleepiness.

Table 2 shows the correlation matrix and the descriptive statistics between the dimensions of MT and objective sleep, subjective sleep and daytime sleepiness across the entire sample $(N=92)$.

$$
\begin{aligned}
= & ============== \\
& \text { Table } 2 \\
== & =============
\end{aligned}
$$

The overall pattern of results shows that any dimension of MT was positively and statistically significantly correlated with sleep efficiency, deep sleep and REM-sleep, and negatively and statistically significantly correlated the number of awakenings after sleep onset, light sleep, subjective sleep complaints and daytime sleepiness. Interestingly, no significant correlations were found for sleep duration and sleep onset latency.

Discussion 
The key findings of the present study are that, in a sample of adolescents, greater mental toughness (MT) was related to better objective sleep, subjective sleep and lower daytime sleepiness. We hold

\section{that the present pattern of results expands the existing}

literature, insofar we found that The present data add to the literature-

\section{in an important way in that we were able to show that greater mental}

toughness (MT) was related to improved objectively assessed sleep among adolescents.

To perform the study, following previous research (Lemola et al $[18,19]$, Gerber et al [9-11]), we expected that higher scores for MT would be associated with improved sleep, as assessed by sleep-EEG recordings, and data did fully confirm this assumption: Adolescents with high MT, relative to adolescents with low $\mathrm{MT}$, had more favorable sleep dimensions such as an increased sleep efficiency, a lower number of awakenings after sleep onset or more deep sleep. Moreover, subjectively, they reported lower sleep complaints (ISI) and a lower score of daytime sleepiness. Insofar, the present data accord with the wealth of studies showing an association between restoring sleep and psychological well-being (cf. 25). Moreover, the present findings mirror previous research in that favorable psychological concepts such as optimism $[18,19]$ were positively related to sleep. However, in our opinion, the present results expand previous findings in that this is the first study to prove that among non-elite sport adolescents, high scores of MT were associated with improved and objectively assessed sleep. Moreover, we also hold that the present data add the literature in an important way in that the construct of mental 
toughness is still poorly investigated, but, in our opinion, has the potential to serve as an umbrella for a broad variety of favorable motivational, emotional, cognitive, and stress-buffering processes. As we could show, higher MT scores were related to stress-resilience and increased physical activity also over time [9-11]. Indeed, research has established links between MT and hardiness, which has previously been found to be associated with stress resilience, and this observation is in accord with the idea that resilience does not evolve from avoidance of adversity, but from successful dealing with negative stimuli [39]. Importantly, stress has been proven to increase the risk for maladjustment and psychopathology during adolescence and early adulthood [5]. In this respect, though highly speculative and not provable with the present data, we assume that MT influences sleep positively via reduced stress [9-11], reduced hyperarousal [29], reduced dysfunctional thoughts [26-28]. Accordingly, it is highly plausible that dysfunctional thoughts and maladaptive behavior are incompatible with the dispositions towards appraisal of high control, challenge, commitment and confidence, that characterize a mentally tough person. Here, specifically and most importantly, this umbrella concept of MT is tightly related to objectively assessed favorable sleep, subjective sleep and lower daytime sleepiness among a sample of nonelite sport adolescents.

Surprisingly, and against expectations, MT was related neither to sleep duration, nor to sleep onset latency. Whereas the relative importance of sleep duration as a key factor for psychological well-being is rather a matter of debate, we have no explanation as for why sleep onset 
latency (SOL) was unrelated to MT: Difficulties to fall asleep is one of the core symptoms of sleep disturbances, and it is assumed that dysfunctional thoughts increase the psycho-physiologic arousal, which in turn hinders to falling asleep [26-29]. Stress as a dysfunctional concept prolongs SOL [29]; MT and stress-resilience are tightly associated [9-11], though, in the present data, no association between MT and SOL could be found.

Despite the clarity of the findings, several considerations warrant against overgeneralization. First, data are derived from a selected subsample, and participants were all recruited only from high schools; therefore a selection bias cannot be excluded. Second, no thorough psychiatric or medical examination was made; therefore, the pattern of results might be due to further latent, though unassessed variables. Third, only participants willing and able complete the questionnaires and to undergo sleep-EEG recordings volunteered to take part in the study; therefore, a positive selection bias cannot be ruled out. Last, in the present model, we assumed that mental toughness as a mental construct does impact on objective sleep, though we are fully aware of the bidirectionality between psychological functioning and sleep; that is, favorable or poor sleep also has an impact on cognitive and emotional processing and behavior (cf. [13]); therefore, it is also conceivable that adolescents sleeping particularly well have developed a particularly high MT.

\section{Conclusions}


Among a sample of participants in their late adolescence, greater mental toughness was associated with an objectively assessed better sleep and low daytime sleepiness. Moreover, it is highly conceivable that improving adolescents' mental toughness may improve also adolescents' sleep. 
References

1 Spear LP. The adolescent brain and age-related behavioral manifestations. Neurosci Biobehav Rev 2000;24:417-63.

2 Pinyerd B, Zipf WB. Puberty - timing is everything! J Pediatr Nurs 2005;20:75-82.

3 Giedd JN, Blumenthal J, Jeffries NO, et al. Brain development during childhood and adolescence: a longitudinal MRI study. Nature Neuroscience 1999;2:861-863.

4 Paus T, Keshavan M, Giedd JN. Why do many psychiatric disorders emerge during adolescence? Nature Rev Neurosci 2008;9:947957.

5 Grant KE, Compas BE, Thurm AE, et al. Stressors and child and adolescent psychopathology: Measurement issues and prospective effects. J Clin Child Adolesc Psychol 2004;33:412-425.

6 Gucciardi DF, Gordon S (Eds.). Mental toughness in sport.

Developments in theory and research. London: Taylor \& Francis, 2011.

7 Crust L, Azadi K. Mental toughness and athletes' use of psychological strategies. Eur J Sport Sci 2012;10:43-51.

8 Jones MI, Parker JK. What is the size of the relationship between global mental toughness and youth experiences? Pers Ind Diff 2013;54:519-523.

9 Gerber M, Kalak N, Lemola S, et al. Adolescents' exercise and physical activity are associated with mental toughness. Phys Activity Mental Health 2012;5:35-42.

10 Gerber M, Kalak N, Lemola S, et al. Are adolescents with high mental toughness levels more resilient against stress? Stress \& Health, in press. 
11 Gerber M, Brand S, Feldmeth AK, et al. Adolescents with high mental toughness adapt better to perceived stress: A longitudinal study with Swiss vocational students. Pers Ind Diff in press.

12 Clough P, Earle K, Sewell D. Mental toughness: The concept and its measurement. In Cockerill I. ed. Solutions in sport psychology. London: Thomson Learning, 2002: 32-46.

13 Haario $\mathrm{P}$, Rahkonen $\mathrm{O}$, Laaksonen $\mathrm{M}$, et al. Bidirectional associations between insomnia symptoms and unhealthy behaviours. J Sleep Res 2013;22:89-95.

14 Owens JA, Spirito A, McGuinn M, Nobile C. Sleep habits and sleep disturbance in elementary school-aged children. J Dev Behav Pediatrics 2000 21, 27-36.

15 Brand S, Kirov R. Sleep and its importance in adolescence and in common adolescent somatic and psychiatric conditions. Int J Gen Med 2011;4:425-442.

16 Colrain I M, Baker FC. Changes in sleep as a function of adolescent development. Neuropsychol Rev 2011;21:5-21.

17 Jan JE, Reiter RJ, Bax MC, et al. Long-term sleep disturbances in children: a cause of neuronal loss. Eur J Paed Neurol 2012;14:380390.

18 Lemola S, Räikkönen K, Scheier MF, et al. Sleep quantity, quality and optimism in children. J Sleep Res 2011;20:12-20.

19 Lemola S, Räikkönen K, Gomez V, Allemand M. Optimism and selfesteem are related to sleep. Results from a large community-based sample. Int J Behav Med, in press.

20 Laberge L, Tremblay RE, Vitaro F, Montplaisir J. Development of parasomnias from childhood to early adolescence. Pediatrics, 2000;106:67-74.

21 Kaneita Y, Ohida T, Osaki Y, et al. Association between mental health status and sleep status among adolescents in Japan: a 
nationwide cross-sectional survey. J Clin Psychiatry 2007;68:14261435.

22 Lund HG, Reider BD, Whiting AB, Prichard JR. Sleep patterns and predictors of disturbed sleep in a large population of college students. J Adolesc Health 2010;46:124-132.

23 Roberts RE, Roberts CR, Duong HT. Chronic insomnia and its negative consequences for health and functioning of adolescents: a 12-month prospective study. J Adolesc Health 2008;42:294-302.

24 Touchette E, Chollet A, Galéra C, et al. Prior sleep problems predict internalising problems later in life. J Affect Dis 2012;143:166-171.

25 Gregory AM, Sadeh A. Sleep, emotional and behavioral difficulties in children and adolescents. Sleep Med Rev 2012;16:129-136.

26 Harvey AG. Pre-sleep cognitive activity: A comparison of sleep-onset insomniacs and good sleepers. Brit J Clin Psychol 2000;39:275-286. 27 Harvey AG. A cognitive model of insomnia. Behav Research Therapy 2002;40:869-894.

28 Carney CE, Edinger JD. Identifying critical beliefs about sleep in primary insomnia. Sleep 2006;29:444-53.

29 Riemann D, Spiegelhalder K, Feige B, et al. The hyperarousal model of insomnia: a review of the concept and its evidence. Sleep Med Rev 2010;14:19-31.

30 Brand S, Gerber M, Kalak N, et al. In adolescence, greater mental toughness is related to better sleep schedules. Behav Sleep Med, under review.

31 Rechtschaffen A. Kales A. A Manual of Standardized Terminology

Techniques and Scoring System for Sleep Stages in Human Subjects. US Department of Health, Education and Welfare, Public Health Service. US Government Printing Office, Washington DC, 1968.

32 Bastien $\mathrm{CH}$, Vallières $\mathrm{A}$, Morin $\mathrm{CM}$. Validation of the Insomnia Severity Index (ISI) as an outcome measure for insomnia research. 
Sleep Med 2001;2:297-307.

33 American Psychiatric Association. Diagnostic and statistical manual of mental disorders. 4th ed. American Psychiatric Association, Washington, DC, 2000.

34 Johns MW. A new method for measuring daytime sleepiness: The Epworth Sleepiness Scale. Sleep, 1991;14:540-545.

35 Masten AS. Regulatory processes, risk, and resilience in adolescent development. Ann NY Acad Sci 2004;1021, 310-319. 